\title{
TECNOLOGÍAS DIGITALES EN CONTEXTOS EDUCATIVOS: UN ANÁLISIS DE LAS COMPETENCIAS DE DOCENTES EN UNA FACULTAD DE EDUCACIÓN, ARTES Y HUMANIDADES EN TIEMPOS DE PANDEMIA
}

\section{Raúl Prada Núñez ${ }^{2}$}

\section{Audin Aloiso Gamboa Suárez ${ }^{3}$ \\ Universidad Francisco de Paula Santander (Colombia).}

\section{RESUMEN}

El presente artículo de investigación pretende analizar las percepciones que tienen los docentes universitarios de una Facultad de

$1 \quad$ Magister en Practicas Pedagógicas, Universidad Francisco de Paula Santander (Colombia). Docente investigador de la Universidad Francisco de Paula Santander. Correo electrónico: erikaalejandrame@ufps.edu.co. Orcid: 0000-0003-1323-8563

2 Docente investigador de la Universidad Francisco de Paula Santander. Magíster en Ingeniería de Análisis de Datos, Mejora de Procesos y Toma de Decisiones por la Universidad Politécnica de Valencia (España). Correo electrónico: raulprada@ufps.edu.co, Orcid: 0000-0001-6145-1786

3 Docente investigador de la Universidad Francisco de Paula Santander. Doctor en Ciencias de la Educación por la Universidad de Cartagena. Correo electrónico: audingamboa@ufps.edu.co, Orcid: 0000-0001-9755-6408
Educación, Artes y Humanidades sobre las competencias digitales en tiempos de crisis que se están viviendo a causa de la pandemia por la COVID - 19. La metodología utilizada se enmarcó en el paradigma cuantitativo descriptivo. Se aplicó un instrumento a 129 docentes para indagar sobre cinco dimensiones de competencias: tecnológicas, pedagógicas, comunicativas, de gestión e investigativas. Los resultados evidencian la necesidad de formación de los docentes para el desarrollo de competencias tecnológicas. Desde sus percepciones, los profesores señalan que hay una debilidad importante en el diseño de 
ambientes de aprendizaje innovadores y el diseño de contenidos digitales que apoyen la práctica pedagógica.

\section{PALABRAS CLAVE:}

Competencias digitales, educación superior, práctica pedagógica, pandemia.

\section{ABSTRAC}

This research article aims to analyze the perceptions that university teachers of a Faculty of Education, Arts and Humanities have about digital skills in times of crisis that are being experienced due to the COVID-19 pandemic. The methodology used is framed in the quantitative descriptive paradigm. An instrument was applied to 129 teachers to inquire about five dimensions of competences: technological, pedagogical, communicative, managerial and investigative. The results show the need for teacher training for the development of technological competencies. From their perceptions, the teachers point out that there is an important weakness in the design of innovative learning environments and the design of digital content that support pedagogical practice.

\section{KEYWORDS:}

Digital skills, higher education, pedagogical practice, pandemic.

\section{INTRODUCCIÓN}

Las competencias han sido consideradas como la respuesta al cambio económico, industrial y laboral que surgió en la década de los setenta, donde es protagonista el método de enseñanza - aprendizaje; adoptando al docente un rol de facilitador y el estudiante sería "responsable de su aprendizaje" (Ruíz, 2006, p. 1). Estas competencias serán entonces una herramienta educativa, como lo afirma Ruiz (2006). Además, este autor, señala que las competencias deben proponerse y conectarse con el ámbito profesional y social en general, el autor afirma que se debe ayudar al estudiante a prepararse para que se inserte en la sociedad como un "profesional ciudadano capaz de resolver problemas en un área determinada" (p. 2) que contribuya a la sociedad en el trabajo conjunto y pueda servirse de los sacrificios ofrecidos para esta de su parte y por los demás individuos (Von Mises, 1980). Dichas competencias, se han caracterizado desde diversos autores por incluir el pensamiento crítico y reflexivo (Gamboa, Montes y Muñoz, 2015), la utilización de las TIC (Hernández Vergel, Prada González, Villabona y Prada Núñez, 2020; Martín, Hernández-Suárez y Mendoza-Lizcano, 2017), el trabajo en grupos heterogéneos, la orientación al aprendizaje (Avendaño, Luna y Gamboa, 2021) la comunicación (Vargas, Gamboa y Prada, 2021), la aplicación del pensamiento matemático y la resolución de problemas (Prada, Gamboa y Hernández, 2021; Hernández, Gamboa y Prada, 2021: Penagos, Mariño y Hernández, 2017)

Por su parte, García (2009) afirma que el discurso de competencias nace del escenario de comunicación e intercambio de experiencias Universidad-egresados-mundo laboral y lo entiende como aquel "lenguaje capaz de responder a la movilidad, la transferencia, el reconocimiento de los aprendizajes y la internacionalización de la Educación Superior" (p. 12). Para García (2009), es necesaria la manifestación transversal-contextual de 4 componentes: "actitudinal, técnico, procedimental y social" (p. 15), observando que "La variedad de matices conceptuales puede observarse al destacar la dimensión originaria, única, de la persona que logra una competencia descifrando las claves del contexto y combinando saberes a través de una síntesis personal" (p. 14), integrando así el saber y el saber ser (García, 2008). A lo anterior podemos agregar el componente de eficacia con base en los cambios obtenidos tras los resultados del 
componente procedimental, que Zabalza (2007) llama saber operar.

A estas dos últimas, Rodríguez (2007) agrega el saber hacer, haciendo del concepto de competencia, un concepto integrado a razón de un Conocimiento teórico o proposicional, derivado de la internalización de afirmaciones empíricas o lógicas sobre el mundo, otro experiencial, también denominado saber del saber estar, del conjunto de normas, valores, actitudes y circunstancias que permiten interactuar con éxito en el medio social y un conocimiento práctico o desarrollo de las habilidades y destrezas necesarias para obrar en el mundo (Tobón, 2006; y, Schwartz, 1994, citados en Rodríguez, 2007, p. 146).

Este autor afirma que, las competencias buscan el aporte de valor al campo laboral específico, desde los recursos humanos sociales y que La nueva visión empresarial considera a la persona como creadora de soluciones óptimas y las organizaciones se convierten en entidades capaces de adaptarse a las circunstancias y facultadas para el aprendizaje. En este panorama, los conocimientos, habilidades y comportamientos de las personas, son las denominadas competencias que deben tener, desarrollar y adquirir los individuos en el mundo de hoy (p. 147).

De manera similar, las competencias pueden ser abordadas como cualificaciones exigidas por el mundo de producción (Castillo y Terrén, 1994), como un componente de la relación salarial, en el que estas - Las relaciones educativas - van a operar en pro de "la socialización para el trabajo asalariado, pero sin olvidar la importancia de "la cualificación como construcción social” (p. 84) o la formación socio-humanística, la formación integral para una calidad de vida, "con crecimiento autosostenido y desarrollo más equitativo" (Rodríguez, 2007, p. 162). Las necesidades en términos de mano de obra asalariada han sido desde un determinado momento el factor más poderoso en la configuración de las políticas educativas y de formación." (p. 76). Castillo y Terrén (1994) advierten que el desfase entre realidad económica o laboral y la educación, si bien es latente, centra su error en la poca o nula indagación sobre la naturaleza de las nuevas necesidades económicas, además de que la planificación educativa no pueda seguir la velocidad de la evolución tecnológica, sino, sobre todo, a la pervivencia de una concepción anacrónica de la cualificación. Una redefinición de esta se presenta como la conditio sine gua non para la necesaria reformulación de la RE en el marco del cambio social actual (Castillo y Terrén, 1994, p. 77).

En concreto, las competencias de los profesores universitarios han sido un factor de amplio interés para los investigadores del campo educativo sobre todo en contextos de la educación superior, en la medida en que se hace latente la necesidad de ser competentes a la hora de enseñar y de esta formar para que los estudiantes adquieran competencias valiosas (Zabalza, 2007); sin embargo, en ocasiones pareciera que para estas instituciones los cambios generados en su contexto han influido lentamente en sus formas de organización y generación del conocimiento (Sesento, 2008).

Estudios como los de Mas y Olmos (2016) han revelado que las competencias en educación superior de los maestros son vistas como punto clave en la búsqueda de calidad y que estas pueden clasificarse en tres secciones a razón de sus alcances en la IES: "El profesor universitario puede desplegar básicamente tres funciones en el desarrollo de su profesión: docencia, investigación y gestión" (p. 439). En el primero se ve al docente como un facilitador (orientador) y a los estudiantes como agentes capaces de buscar, procesar y aplicar el conocimiento; saber seguir estos tres pasos (Tomás, 2001, citado en Mas y Olmos, 2016). En segundo lugar, se menciona "la investigación competitiva, creación 
de equipos multidisciplinares integrados, a su vez, por miembros de diferentes universidades, países, etcétera" (Tomás, 2001, citado en Mas y Olmos, 2016, p. 440). El tercero se refiere a la participación opcional y la disposición del profesor en la gestión de la Universidad.

Ahora bien, según los precedentes autores, se hace necesario para entender dichas competencias, el diferenciar - A pesar de la interconexión e interrelación - los tres escenarios de actuación de los docentes universitarios: el micro contexto de aula, donde investigar sobre su propia profesión y la gestión de los recursos; el escenario institucional, donde la investigación acompaña la coordinación y la gestión como organización y, el escenario sociolaboral y cultural, donde la investigación gira en torno al "desarrollo de convenios, transferencia tecnológica con instituciones externas, aunque también están presentes la gestión y docencia referentes a las relaciones y actividades extrainstitucionales."( Mas y Olmos , 2016, p. 441).

Posterior a esto, Mas y Olmos entonces (2006) señalan que las competencias del profesional en docencia universitaria tienen unas funciones tales como:

1.) Diseñar una guía docente que se acople a las necesidades surgidas por los actores académicos, al contexto en que se encuentre, para formar de allí su perfil profesional y sus objetivos a alcanzar como formador y al legado académico, disciplinar, metodológico, legal, etc., en torno a su orientación profesional dentro de la IES; 2.) Desarrollar el proceso de enseñanzaaprendizaje, propiciando oportunidades de aprendizaje tanto individual como grupal; 3.) Tutorizar el proceso de aprendizaje del alumno propiciando acciones que le permitan una mayor autonomía; 4.) Evaluar el proceso de enseñanza-aprendizaje; 5.) Contribuir activamente a la mejora de la calidad de la docencia; 6.) Participar activamente en la dinámica académico-organizativa de la institución (universidad, facultad, departamento, unidad $\mathrm{a}$ área, titulaciones, etc.) (p. 446).

En lo que corresponde a la formación de docentes, la investigación de Gallego et al (2010), afirman, desde la percepción recogida por los futuros docentes, que las competencias en esta profesión, se desarrollan mediante una "fusión entre aspectos teóricos y prácticos, combinando ambos en un quehacer diario motivado" (p. 16). Dichos autores recalcan que aquel reto debe responder a la demanda de "la sociedad de la información y el conocimiento, la convergencia hacia el Espacio Europeo de Educación Superior y la reforma de las titulaciones" (p. 16). Los autores dirigen su mirada hacia la importancia de desarrollar estas competencias tecnológicas, afirmando que "cuando se trata de la competencia tecnológica del futuro docente, es importante advertir que nos encontramos con estudiantes con capacidades bien distintas, asociadas por lo general a sus actitudes hacia las TIC" (p. 2). Gallego et al (2010), cierran su investigación con la afirmación de que si bien él da educación básica secundaria se debe adquirir una competencia digital, la formación universitaria docente debe incluir su abordaje, ya que esta, además de comportarse como una herramienta para la resolución eficiente de problemas reales, posibilita evaluar y seleccionar nuevas fuentes de información e innovaciones tecnológicas a medida que van apareciendo, en función de su utilidad para acometer tareas $u$ objetivos específicos" (p. 16).

La presente investigación pretende realizar una lectura a las competencias digitales de los profesores universitarios, campo de estudio que cobra una importancia relevante por los tiempos de crisis que se están viviendo a causa de la 
pandemia por la COVID -19. En este sentido, Rambay y De la Cruz (2020), desde Ecuador, afirman que "se autorizó que las universidades ajusten las actividades de aprendizaje para que puedan ser desarrolladas e impartidas mediante el uso de tecnologías interactivas multimedia y entornos virtuales de aprendizaje, a través de plataformas digitales" (p. 512) a razón de la mencionada enfermedad, se proponen realizar una revisión de evidencias científicas de las competencias que han desarrollado los docentes universitarios en el contexto actual de pandemia.

Dicha búsqueda de evidencias se da con apoyo de tres bases de datos y un aplicativo web, pudiendo concluir de esta forma que es necesario fortalecer las competencias del rol docente, la creación de contenido digital y la seguridad; competencias que clasifican en tres: creatividad, trabajo colaborativo y alfabetización digital. "Los entornos virtuales, las herramientas tecnológicas, y las aplicaciones con enfoque académico, se han convertido en medios para desarrollar dichas competencias" (Rambay y De la Cruz 2020, p. 512).

También Laurente et al (2020), desde Lima en su estudio desarrollo de competencias digitales en docentes universitarios a través de entornos virtuales: experiencias de docentes universitarios en Lima", orientan su búsqueda académica en descubrir cómo desenvolverse en entornos virtuales, mediante habilidades y conocimientos, para permitir, desde estos espacios, el desarrollo de competencias digitales. Laurente et al (2020) utilizan la revisión documental como instrumento para apoyar su metodología mixta de tipo concurrente. Sus resultados reflejan la innovación y la investigación como puntos clave en los aportes de la combinación entre presencialidad y entornos virtuales, por lo que concluyen reafirmando el reto que supone el panorama actual para Universidades y docentes y sugiriendo para el desarrollo de competencias de docentes universitarios "el conocimiento, las habilidades y uso responsable de las tecnologías digitales" (p. 2).

Por su parte, Sandi y Sanz (2018), se encaminan a indagar sobre propuestas hechas en torno a dimensiones e indicadores que se requieren en la docencia de educación superior de Iberoamérica, por medio de una revisión documental, que permitió dilucidar tres resultados principales en dicha investigación: obtención de conocimientos tecnológicos, pedagógicos y disciplinares; la influencia recíproca existente entre competencias pedagógicas y tecnológicas y, la ampliación del alcance de las competencias tecnológicas hacia otros espacios de actuación docente como lo son la gestión administrativa y la investigación.

Tejada y Pozos (2018), invitan a una redefinición del perfil profesional y con ella a las competencias profesionales de docentes, que se adapten a los nuevos escenarios motivados por las TIC, hablan entonces de la profundización y el sentido de las competencias digitales. Los autores reparan además en las nuevas implicaciones para los formadores de docentes, dentro de las que cabe mencionar la educación continua, el trabajo en equipo, la correspondencia entre currículo y competencias, vinculación con la vida real y el trabajo (praxis), etc.; y, busca integrar dichas competencias en el perfil del docente universitario. Se concluye tras esta investigación que se hace necesario definir el grado de dominio que se tenga sobre cierta competencia, a razón de un aprendizaje permanente y que la integración de la Competencia Digital del profesor ha de estructurarse en tres grandes fases (competencias básicas, de profundización y de generación del conocimiento), concebidas como un proceso complejo en el que el profesorado va profundizando en el conocimiento y uso de las TIC y las va integrando gradual y significativamente en su actividad docente a través de la reflexión crítica, como elemento 
esencial en todo este proceso (Pozos, 2010, citado en Tejada y Pozos, 2018, p. 36).

Finalmente, Cabero et al (2020), brinda un aporte teórico sustancial en torno a un marco de competencias limadas desde su objetivo de contribuir a ampliar dicha investigación hacia el diseño, producción y evaluación de T.MOOC para la adquisición por los docentes de Competencias digitales docentes. Estos autores remarcan, citando a Fernández et al (2017), que es por falta de apoyo institucional, de recursos y la existencia nula de planes de formación, que los docentes no se forman en TIC para la educación. Así mismo, cabe resaltar según los autores la incidencia de las competencias del Marco Europeo de Competencia Digital del Profesorado (Unión Europea), las del Marco Común de Competencia Digital Docente del Intef (España) y las Competencias TIC para el Desarrollo Profesional Docente (Colombia)

\section{METODOLOGÍA}

A continuación se desglosan las principales características de la investigación realiza con docentes de la Facultad de Educación, Artes y Humanidades de una universidad pública del nororiente colombiano, cuyo objetivo era diagnosticar el nivel de apropiación de las competencias digitales de los docentes después de un año de estar trabajando en modalidad no presencial a causa de la pandemia del COVID-19 que obligó a un aislamiento social como posible herramienta para frenar la expansión de esta enfermedad en la población.

\section{CARACTERÍSTICAS DE LA INVESTIGACIÓN}

Los datos para esta investigación son recolectados en un momento específico de tiempo que corresponde a exactamente trece meses después de la declaratoria de aislamiento social, situación que obligó al sistema educativo a cambiar de paradigma de enseñanza, con el fin de garantizar el servicio educativo.
En la institución de educación superior objeto de esta investigación desde marzo de 2020 se inició el trabajo de enseñanza bajo un enfoque no presencial asistido con diversidad de recursos TIC. Razón por la cual los datos son recolectados directamente de los docentes adscritos a la Facultad de Educación, Artes y Humanidades y que orientan asignaturas en algunos de los siete programas de formación profesional de la Facultad.

Los datos recolectados se procesaron de forma descriptiva con el fin de obtener una serie de estadísticos que facilitaran la caracterización de los informantes alrededor del objetivo de investigación. Por lo anterior, se concluye que esta investigación se clasifica dentro del enfoque cuantitativo a nivel descriptivo transversal con diseño de campo (Avendaño, Gamboa y Prada, 2021).

\section{POBLACIÓN Y MUESTRA}

La población para esta investigación corresponde a la totalidad de docentes que durante el primer semestre del 2021 orientaron alguna asignatura de la oferta académica de la Facultad de Educación, Artes y Humanidades, en ellos se incluyen tres tipos de vinculación laboral: docentes de planta, docentes ocasionales y docentes catedráticos.

Para la selección de la muestra se recurre al muestreo no probabilístico bajo la técnica de muestreo voluntario, puesto que a la totalidad de docentes se les envió mediante correo electrónico una invitación a diligenciar el instrumento por medio del enlace de la versión en línea del mismo.

Los docentes accedieron de forma libre y voluntaria al instrumento hasta completar un total de 129 personas, después de tres semanas de ventana de tiempo para la recolección de datos. Este tamaño de muestra representa 
aproximadamente el $69.7 \%$ del tamaño de la población.

\section{INSTRUMENTO DE RECOLECCIÓN DE DATOS}

Para alcanzar el objetivo en esta investigación se diseña un instrumento en el que inicialmente se hace una caracterización del perfil demográfico y profesional de cada docente, para posteriormente diagnosticar el nivel de dominio y apropiación pedagógica de las competencias digitales que poseen los profesores de la Facultad de Educación, Artes y Humanidades después de más de un año de estar laborando en un modelo de enseñanza no presencial asistido por recursos TIC.

Se analizan cinco dimensiones de competencias (tecnológicas, pedagógicas, comunicativas, de gestión e investigativas) y al interior de cada una de ellas se subdivide en tres sub dimensiones, siendo coherente con el Modelo de Competencias TIC propuesto por el Ministerio de Educación Nacional de Colombia.

De esta forma se conforma un cuestionario con 93 reactivos, de ellos 8 corresponde al perfil demográfico y los 85 restantes a las competencias. En todos los casos se formularon opciones de respuesta cerradas y en caso particular de las competencias, se aplicó una escala de valoración Likert con cinco niveles de aprobación.

Dado que se realizaron algunos ajustes en cuanto a la redacción de los ítems, se procedió a determinar el análisis de fiabilidad en función del constructo general del instrumento $y$ desagregado por cada nivel de competencia (ver Tabla 1).

Tabla 1. Estadísticos de fiabilidad

\begin{tabular}{lcc}
\hline \multicolumn{1}{c}{ Constructo analizado } & Alfa de Cronbach & $\mathbf{N}^{\circ}$ de elementos \\
\hline Competencias Digitales de los docentes & 0.991 & 85 \\
\hline Competencias Tecnológicas & 0.962 & 15 \\
\hline Competencias Pedagógicas & 0.965 & 17 \\
\hline Competencias Comunicativas & 0.955 & 16 \\
\hline Competencias de Gestión & 0.982 & 19 \\
\hline Competencias Investigativas & 0.975 & 18 \\
\hline
\end{tabular}

A partir de los valores obtenidos para el estadístico Alfa de Cronbach en cada uno de los constructos de análisis, se puede concluir que los ítems considerados son pertinentes para medir los constructos que se están investigando y según Montgomery y Runger (2010) la escala ofrece un nivel satisfactorio de fiabilidad.

\section{PROCESAMIENTO DE LOS DATOS}

Una vez los datos son recolectados cumplida la ventana de tiempo destinada a esta actividad, se procedió a descargar del Google Form el archivo de Excel con el fin de revisar la posible existencia de datos atípicos, para avanzar a la codificación de los datos que posteriormente son exportados al software SPSS v25 con el fin de ser procesados desde la estadística descriptiva. Al final los resultados son organizados y representados en Excel.

\section{RESULTADOS}

Los resultados son organizados y presentados en la forma en que aparecen en el instrumento de recolección de datos. 


\section{PERFIL DE LOS DOCENTES}

A través de los descriptivos mostrados en la Tabla 2 se puede resaltar que existe ligero predominio del género femenino entre los docentes encuestados, donde el $54.7 \%$ de ellos tienen edades que oscilan entre los $31 \mathrm{y}$ 50 años, de ellos el $57.3 \%$ poseen una relación estable mientras que el $31.8 \%$ son solteros. Los docentes del Departamento académico de Ciencias Humanas, Sociales e Idiomas fueron los que más apoyaron con el diligenciamiento de la encuesta, entre tanto el $14.0 \%$ laboran en programas de la Facultad, pero provienen de otros departamentos académicos. Al explorar sobre el programa académico en el que concentran la mayor parte de su carga laboral, se identificó que el $65.1 \%$ de ellos laboran en los programas de Trabajo Social, Arquitectura y Derecho, situación que resulta coherente dado son los programas que concentran más de la mitad de los estudiantes matriculados en la Facultad. Con relación a la formación posgradual alcanzada por los docentes, se determinó que el $64.3 \%$ cuentan con estudios de Maestría. Finalmente, el $82.2 \%$ de los docentes de la muestra están vinculados laboralmente con la institución educativa por medio de hora cátedra.

Tabla 2. Descriptivos asociados con el perfil de los docentes

\begin{tabular}{|c|c|c|c|}
\hline Variable & Niveles de respuesta & Frecuencia & Porcentaje \\
\hline \multirow{3}{*}{ Género } & Femenino & 68 & 52.7 \\
\hline & Masculino & 61 & 47.3 \\
\hline & Total & 129 & 100.0 \\
\hline \multirow{6}{*}{ Rango de edad } & Entre 20 y 30 años & 11 & 8.5 \\
\hline & Entre 31 y 40 años & 37 & 28.7 \\
\hline & Entre 41 y 50 años & 37 & 28.7 \\
\hline & Entre 51 y 60 años & 29 & 22.5 \\
\hline & Más de 60 años & 15 & 11.6 \\
\hline & Total & 129 & 100.0 \\
\hline \multirow{6}{*}{ Estado civil } & Casado(a) & 59 & 45.7 \\
\hline & Separado(a) & 12 & 9.3 \\
\hline & Soltero(a) & 41 & 31.8 \\
\hline & Unión libre & 15 & 11.6 \\
\hline & Viudo(a) & 2 & 1.6 \\
\hline & Total & 129 & 100.0 \\
\hline \multirow{6}{*}{$\begin{array}{l}\text { Departamento } \\
\text { académico en el que } \\
\text { está adscrito }\end{array}$} & Arquitectura y Urbanismo & 23 & 17.8 \\
\hline & Ciencias Humanas, Sociales e Idiomas & 54 & 41.9 \\
\hline & Derecho & 21 & 16.3 \\
\hline & $\begin{array}{l}\text { Pedagogía, Andragogía, Comunicación y } \\
\text { Multimedios }\end{array}$ & 13 & 10.1 \\
\hline & Otro departamento & 18 & 14.0 \\
\hline & Total & 129 & 100.0 \\
\hline
\end{tabular}




\begin{tabular}{|c|c|c|c|}
\hline \multirow{8}{*}{$\begin{array}{l}\text { Programa académico } \\
\text { en el que tiene la } \\
\text { mayor parte de su } \\
\text { carga académica }\end{array}$} & Arquitectura & 24 & 18.6 \\
\hline & Comunicación Social & 7 & 5.4 \\
\hline & Derecho & 20 & 15.5 \\
\hline & Lic. En Ciencias Naturales & 11 & 8.5 \\
\hline & Lic. En Educación Infantil & 11 & 8.5 \\
\hline & Lic. En Matemáticas & 16 & 12.4 \\
\hline & Trabajo Social & 40 & 31.0 \\
\hline & Total & 129 & 100.0 \\
\hline \multirow{5}{*}{$\begin{array}{l}\text { Máximo nivel de } \\
\text { escolaridad }\end{array}$} & Posdoctorado & 3 & 2.3 \\
\hline & Doctorado & 8 & 6.2 \\
\hline & Maestría & 83 & 64.3 \\
\hline & Especialización & 35 & 27.1 \\
\hline & Total & 129 & 100.0 \\
\hline \multirow{4}{*}{$\begin{array}{c}\text { Tipo de vinculación } \\
\text { laboral }\end{array}$} & Planta tiempo completo & 4 & 3.1 \\
\hline & Ocasional & 19 & 14.7 \\
\hline & Catedrático & 106 & 82.2 \\
\hline & Total & 129 & 100.0 \\
\hline
\end{tabular}

\section{COMPETENCIAS DIGITALES}

Después de más de doce meses de trabajo en modalidad no presencial con apoyo en recursos TIC, es de vital importancia para la Decanatura de la Facultad identificar las necesidades de cualificación de los docentes que laboran en la Facultad con el fin de mejorar la calidad de sus prácticas pedagógicas.

En la Tabla 3 se puede identificar que menos del $5.0 \%$ de los docentes adscritos a los departamentos de Derecho, Arquitectura y
Urbanismo presentan desempeños bajos a nivel general en cuanto a las cinco competencias digitales definidas por el Modelo del MinEducación de Colombia. Así mismo, se puede destacar que en promedio el $38.0 \%$ presentan desempeños medios, es decir, que en este tiempo han desarrollado ciertas competencias digitales, pero requieren robustecer en otro aspecto. Finalmente, se reseña que en promedio seis de cada diez docentes que diligenciaron el cuestionario aseguran poseer desempeños altos en cuanto los ítems evaluados en las cinco dimensiones de las competencias digitales.

Tabla 3. Comparativo del desempeño de las competencias digitales de los docentes desagregado por Departamento Académico

\begin{tabular}{|c|c|c|c|c|}
\hline \multirow[b]{2}{*}{ Departamento académico } & \multicolumn{3}{|c|}{ Competencias Digitales } & \multirow[b]{2}{*}{ Total } \\
\hline & Desempeño Bajo & Desempeño Medio & $\begin{array}{l}\text { Desempeño } \\
\text { Alto }\end{array}$ & \\
\hline Arquitectura y Urbanismo & $4,3 \%$ & $26,1 \%$ & $69,6 \%$ & $100,0 \%$ \\
\hline $\begin{array}{l}\text { Ciencias Humanas, Sociales e } \\
\text { Idiomas }\end{array}$ & & $35,2 \%$ & $64,8 \%$ & $100,0 \%$ \\
\hline Derecho & $4,8 \%$ & $52,4 \%$ & $42,9 \%$ & $100,0 \%$ \\
\hline $\begin{array}{l}\text { Pedagogía, Andragogía, } \\
\text { Comunicación y Multimedios }\end{array}$ & & $50,0 \%$ & $50,0 \%$ & $100,0 \%$ \\
\hline Otro departamento & & $30,8 \%$ & $69,2 \%$ & $100,0 \%$ \\
\hline Total & $1,6 \%$ & $38,0 \%$ & $60,5 \%$ & $100,0 \%$ \\
\hline
\end{tabular}


Por medio de la Gráfica 1 se puede concluir que no existen diferencias significativas entre la puntuación media alcanzada por los docentes de los diversos departamentos académicos con respecto al Modelo de Competencias Digitales propuesto por el MinEducación. Por tal motivo, en adelante no se establecerán diferencias entre ellos.

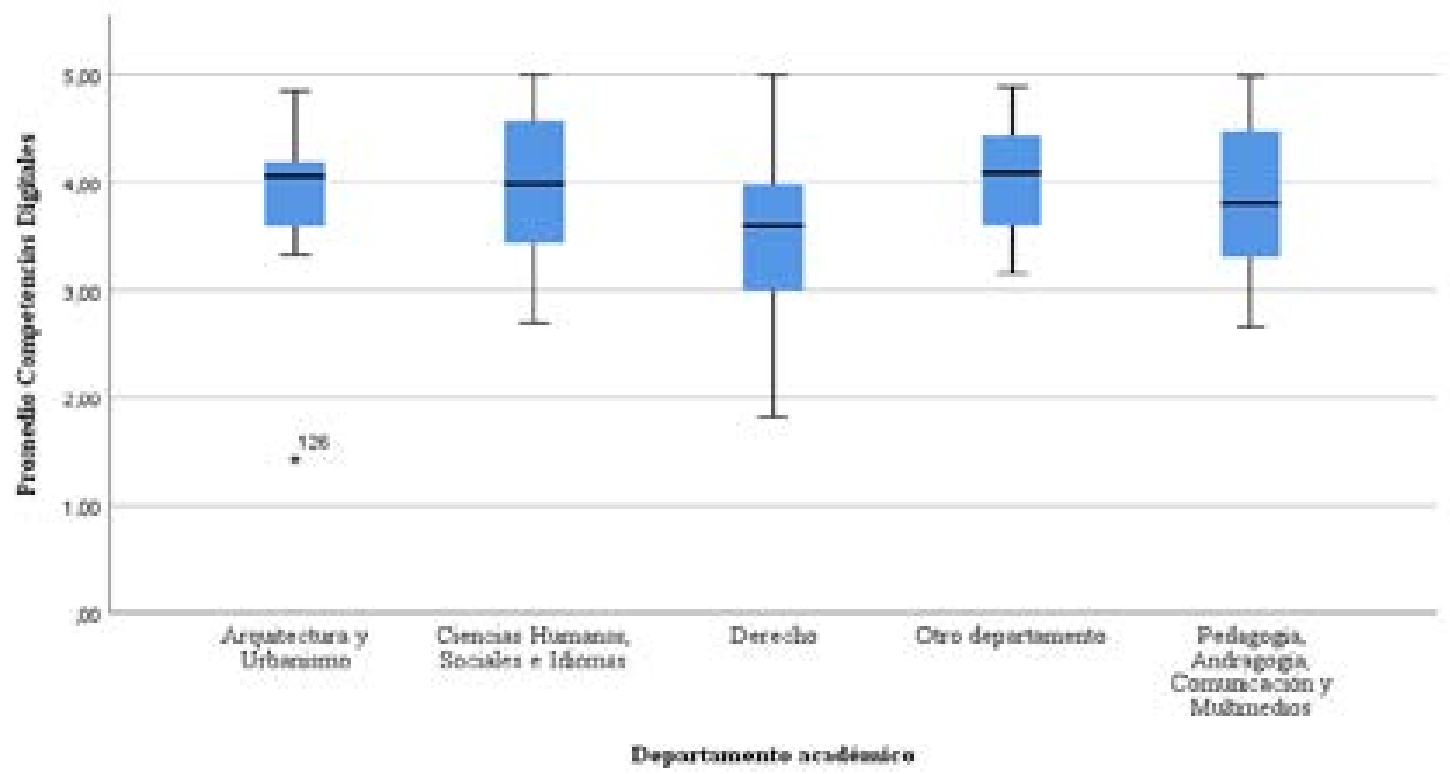

Gráfica 1. Diagrama de cajas y bigote comparativo del promedio de la puntuación a nivel general de las competencias digitales desagregadas por Departamento Académico

Por medio de la Tabla 4, se puede comparar el nivel de desempeño manifiesto por los docentes respecto a las diversas competencias consideradas, en contraste con el promedio de cada una ellas. Apoyados en el promedio se puede observar que las competencias de Gestión son las que requieren mayor refuerzo, entre tanto las Pedagógicas y las Comunicativas se muestran como un desempeño alto que supera el $65 \%$ de los casos observados.

Tabla 4. Comparativo de los descriptivos para cada una de las competencias analizadas

\begin{tabular}{|c|c|c|c|c|}
\hline \multirow{2}{*}{ Competencias } & \multicolumn{3}{c}{ Nivel de desempeño } & \multirow{2}{*}{ Promedio } \\
\cline { 2 - 5 } & $1.6 \%$ & $42.6 \%$ & $55.8 \%$ & 3.84 \\
\hline Tecnológicas & $0.8 \%$ & $27.1 \%$ & $72.1 \%$ & 4.09 \\
\hline Pedagógicas & $1.6 \%$ & $33.3 \%$ & $65.1 \%$ & 4.00 \\
\hline Comunicativas & $3.1 \%$ & $48.1 \%$ & $48 . .8 \%$ & 3.68 \\
\hline De Gestión & $1.6 \%$ & $41.9 \%$ & $56.6 \%$ & 3.80 \\
\hline Investigativas & &
\end{tabular}


A continuación, se desagregan cada una de las dimensiones con el fin de identificar aquellos aspectos por mejorar o para robustecer en el proceso de mejoramiento pedagógico y tecnológico de los docentes.

\section{COMPETENCIAS TECNOLÓGICAS}

Estas competencias pretenden analizar la selección y uso pertinente y eficiente de las diversas herramientas tecnológicas, la forma como el docente las combina con la finalidad de potenciar su uso en el contexto académico. Se observa que en promedio el $95.5 \%$ de los docentes aseguran que son muy competentes en el conocimiento de las diversas herramientas tecnológicas y su integración en la práctica educativa.

Con respecto a las diversas herramientas tecnológicas en los procesos educativos y su coherencia con el área de formación, el nivel y contexto de los estudiantes; se pudo determinar que el $25.8 \%$ de los docentes encuestados aseguran que no son competentes en el diseño de contenidos digitales que apoyen el proceso de aprendizaje a partir de la adecuada implementación de las diversas herramientas tecnológicas de que dispone tanto en la institución como del medio en general.

Al indagar sobre el diseño de ambientes de aprendizaje que sean innovadores, se determinó que en promedio el $36.4 \%$ de los docentes de la Facultad, aseguran que requieren de capacitación en el proceso de diseño de entornos virtuales de aprendizaje tendientes a fortalecer el desarrollo de las competencias de sus estudiantes tal como se reseña en la investigación de López-García y GutiérrezNiño (2018), desconociendo la conformación de comunidades o redes que apoyen y garanticen procesos de aprendizaje significativo.

\section{COMPETENCIAS PEDAGÓGICAS}

Con estas competencias se desea que el docente robustezca los procesos tanto de enseñanza como de aprendizaje, con una visión clara de las limitaciones y los alcances que trae en el desarrollo profesional y en la formación de los estudiantes la incorporación de los recursos tecnológicos. En este sentido se identificó que aproximadamente el $74.4 \%$ de los docentes aseguran ser altamente competentes debido a que realizan un proceso reflexivo permanente sobre su práctica pedagógica, identificando los problemas educativos con el fin focalizar su práctica en solventar estas dificultades, al tiempo que reconoce en la labor pedagógica un constante aprendizaje que demanda en algunos casos de tomar la iniciativa para la realización de procesos de autoaprendizaje. En esta categoría de análisis, se observa como un aspecto a mejorar en el $25.6 \%$ de los docentes, quienes reconocen que requieren robustecer la formulación e incorporación de proyectos educativos por medio de los cuales los estudiantes reflexionen sobre la producción de conocimiento que propenda por un verdadero aprendizaje, apoyado en el trabajo colaborativo, las TIC y en algunos casos, en el trabajo autónomo.

Se resalta como una fortaleza del proceso de enseñanza en opinión de al menos el 90.0\% de los docentes de la Facultad de Educación, aseguran que en su trabajo de aula siempre establecen conexiones entre los temas que desarrollan y los problemas cotidianos que viven los estudiantes en su vida. En este sentido Villamizar-Acevedo, Lozano-león y Sierra-Garavito (2017) afirman esto estímula al proceso de aprendizaje, puesto que les ofrece un espacio de aplicabilidad de los saberes de la disciplina.

Cuando se explora en los docentes la generación de experiencias significativas que involucren ambientes de aprendizaje en los que se 
reconocen las diferencias en cuanto a los ritmos de aprendizaje, el $38.8 \%$ de ellos aseguran que necesitan instrucción en este aspecto mientras que el porcentaje restante se muestran seguros de poseer estas competencias, ya que manifiestan que frecuentemente diseñan ambientes de aprendizaje mediados por TIC con el fin de propiciar el desarrollo integral de estudiantes en campos como el cognitivo, el psicológico, el físico y el social, entre muchos otros.

\section{COMPETENCIAS DE COMUNICACIÓN}

Por medio de estas competencias se pretende expresar, establecer contacto y relacionarse en espacios virtuales y audiovisuales a través de diversos medios y con el manejo de múltiples lenguajes, de manera sincrónica y asincrónica. Respecto a los canales de comunicación utilizados en estos semestres de trabajo no presencial, el $96.9 \%$ de los docentes que laboran en los programas de la Facultad de Educación aseguran que han habilitado diversidad de canales de comunicación con los estudiantes con la finalidad de facilitar tanto el trabajo sincrónico de las clases, asesorías y demás actividades, como el asincrónico en cuanto el desarrollo de actividades de formación complementarias.

Reconocen como una fortaleza en este tiempo las habilidades que han ido desarrollando para maximizar los beneficios que ofrece la internet, puesto que les ha permitido vincularse de forma más sencilla a diversas redes de docentes o investigadores con quienes comparten intereses comunes, al tiempo que motivan a sus estudiantes a ser parte activa de estas comunidades también.

El $27.9 \%$ de los docentes reconocen que necesitan de orientación en el proceso de diseño de intervenciones pedagógicas en las que se combinen diversos recursos como texto, audio, videos, imágenes tanto dinámicas como estáticas, que faciliten el entendimiento de los saberes propios de cada asignatura.

\section{COMPETENCIAS DE GESTIÓN DE LOS DOCENTES.}

Por medio de estas competencias se busca evaluar todos los procesos propios de la actividad pedagógica como son la planeación, organización, administración y evaluación de forma efectiva tanto las prácticas pedagógicas como el desarrollo institucional con el apoyo de las TIC. En el $92.3 \%$ de los casos, los docentes reconocen que son competentes en lo concerniente con el conocimiento de políticas que regulan la privacidad durante el uso de recursos TIC, pero en ese mismo porcentaje existe desconocimiento en cuanto a las políticas relacionadas con el impacto ambiental y los riesgos a la salud que se derivan del uso de los recursos TIC.

El $13.2 \%$ de los docentes encuestados aseguran que dentro del desarrollo de su proceso pedagógico con los estudiantes nunca han propuesto ni han desarrollado procesos de mejoramiento y seguimiento del uso de las TIC en la gestión de los cursos. El $38.0 \%$ del grupo manifiestan que no han seleccionado ni han accedido a programas de formación que satisfagan sus necesidades de desarrollo profesional que respondan a las exigencias actuales en cuanto al uso de recursos TIC.

El 91.5\% manifiestan que en sus cursos han evaluado los beneficios de las herramientas TIC en diversos campos como lo son la gestión curricular, la proyección social y el vínculo con el Proyecto Educativo Institucional. Pero en lo que nunca han trabajado es en el desarrollo de políticas escolares o institucionales tendientes a regular la privacidad, el impacto ambiental y la salud de los estudiantes. 


\section{COMPETENCIAS INVESTIGATIVAS}

Por medio de estas competencias se espera que los docentes, haciendo uso de los diversos recursos TIC, transformen sus procesos de investigación formativa y la generación de nuevo conocimiento en su campo de desempeño. Se evalúa como primer aspecto, el registro y seguimiento de lo realizado con los estudiantes utilizando las TIC. En este sentido se determinó que el $89.9 \%$ de los docentes aseguran que por medio del uso de la plataforma institucional han documentado el trabajo realizado con los estudiantes, tales como actividades de clase, observaciones de talleres, paneles de discusión, consultas en bases de datos, consultas en otras fuentes de información para la validación de la calidad de la información y demás espacios de formación que se han considerado en el curso.

En lo que respecta a la formulación y elaboración de proyectos de investigación colaborativa en los que participan de forma activa los estudiantes junto con el docente, este mismo porcentaje de docentes aseguran que han orientado a sus estudiantes en el uso de plataformas especializadas de carácter académico, acompañado de orientaciones para la organización, representación e interpretación de información derivada de procesos de micro investigaciones adelantadas desde cada curso.

Finalmente, se exploró en los docentes las habilidades y estrategias educativas clasificadas como innovadoras cuyo principal objetivo es la generación colectiva de conocimiento y su respectiva difusión. En este sentido aproximadamente el $20.2 \%$ de los docentes encuestados que laboran en la Facultad de Educación han resaltado la necesidad de recibir orientación en actividades como la selección de mecanismo de divulgación, la participación en redes académicas especializadas y/o la participación en proyectos colaborativos que habitualmente se derivan de las redes académicas, en las que participen de forma activa con estudiantes y docentes a la generación colectiva de nuevo conocimiento.

\section{CONCLUSIONES Y DISCUSIÓN}

El estudio logra evidenciar que durante el aislamiento causado por la pandemia de la Covid -19 , la gran mayoría de los docentes han logrado desarrollar competencias digitales que en tiempos de presencialidad no habían explorado. Estas evidencias abren una reflexión importante sobre la formación en competencias digitales de los futuros profesores como lo evidencian los estudios de Ibañez (2020), Gamboa, Hernández y Prada (2021) y Avendaño, Gamboa y Prada, (2021)

Un elemento importante que es necesario resaltar, es la necesidad de formación que señalan los docentes para el desarrollo de competencias tecnológicas. Desde sus percepciones, los docentes señalan que hay una debilidad importante en el diseño de ambientes de aprendizaje innovadores y el diseño de contenidos digitales que apoyen la práctica pedagógica. Estos resultados se pueden contrastar con los estudios de Ardila (2009) y Prada, Hernández y Gamboa (2019) quienes afirman que se hace necesario replantear los currículos en los programas de formación de maestros y fortalecer el desarrollo de competencias tecnológicas y digitales visualizando la inevitable virtualización de la educación superior.

Con relación a las competencias pedagógicas de los docentes en esta modalidad de educación asistida por las tecnologías digitales, se logró evidenciar espacios de reflexión y autoevaluación de la propia práctica del profesor, que según la opinión de los mismos docente ha llevado a que se transforme su propia práctica pedagógica. En este sentido, se puede citar a Barragán (2015), cuando categóricamente señala que la complejidad de una práctica como la del maestro, expuesto a las transformaciones 
contemporáneas, lo ha obligado a encontrar caminos seguros y estables para la enseñanza, la educación y la formación en tiempos revueltos como estos de la cibercultura (p.57).

Por último, es importante resaltar las habilidades que los profesores han adquirido para relacionarse con docentes e investigadores en los ámbitos nacionales e internacionales usando las tecnologías digitales, elementos que no se evidenciaban recurrentemente antes de la pandemia. Esta nueva cultura de interacción digital, sin duda, fortalece de una manera significativa, no solo el desarrollo de competencias digitales, sino la investigación y la internacionalización de la misma.

\section{REFERENCIAS BIBLIOGRÁFICAS}

Ardila Rodríguez, Mireya. (2009). Docencia en ambientes virtuales: nuevos roles y funciones. Revista Virtual Universidad Católica del Norte, (28). Recuperado http://revistavirtual.ucn.edu.co/index. php/RevistaUCN/article/view/82

Avendaño, W. R., Gamboa, A. A., \& Prada, R. P. (2021). Diseño de una escala para la evaluación de las percepciones de actores educativos sobre los programas de licenciatura en Colombia. Revista Boletín Redipe, 10(5), 323-331. Doi: https://doi.org/10.36260/rbr.v10i5.1307

Avendaño-Castro, W. R., Luna-Pereira, H. O., \& Gamboa-Suárez, A. A. (2021). Estilos de aprendizaje en educación superior: lecturas desde un programa de ciencias empresariales en una universidad pública. Saber, Ciencia y Libertad, 16(1). Doi: $\quad$ https://doi.org/10.18041/23823240/saber.2021v16n1.7528

Avendaño, W. R., Gamboa, A. A., \& Prada, R. (2021). Jaque a la educación: percepciones de maestros en formación de una universidad pública sobre la calidad de la enseñanza en tiempos de crisis. Revista Boletín Redipe, 10(5), 71-82. Recuperado de: https://doi. org/10.36260/rbr.v10i5.1286

Cabero, J., Barroso, J., Palacios, A. y Llorente, C. (2020). Marcos de Competencias Digitales para docentes universitarios: su evaluación a través del coeficiente competencia experta. Revista Electrónica Interuniversitaria de Formación del Profesorado, 23(3), 17-34. https://doi.org/10.6018/ reifop. 414501

Castillo, C., y Terrén, E. (1994). De la cualificación a la competencia: elementos para una reconstrucción epistemológica. Cuadernos de relaciones laborales, (4), 75-91. Recuperado de: https://dialnet.unirioja. es/servlet/articulo?codigo $=165222$

Giraldo, D. F. B. (2015). Cibercultura y prácticas de los profesores: entre hermenéutica y educación. Universidad de la Salle.

Gamboa, A., Montes, A., \& Muñoz, P. (2015). Participación crítica y democrática: comprensión de los discursos de actores educativos. Zona próxima: revista del Instituto de Estudios Superiores en Educación, (22), 5668. Recuperado de: http://www. scielo.org.co/scielo. php?pid=S0124$71072017000100060 \&$ script $=s c i$ abstract\&tlng=en

Gallego, M.J., Gámiz, V., \% Gutiérrez, E. (2010). El futuro docente ante las competencias en el uso de las tecnologías de la información y comunicación para enseñar. EDUTEC, Revista Electrónica de Tecnología Educativa (34). Recuperado: http:// edutec.rediris.es/revelec2/revelec34/ 
García San Pedro, M.J. (2008). Realidad y perspectivas de la formación por competencias en la universidad. (Tesis Doctoral). Universitat Autònoma de Barcelona.

García San Pedro, M.J. (2009). El Concepto de Competencias y su Adopción en el Contexto Universitario. Revista Alternativas. Cuadernos de Trabajo Social, (16). 11- 28. Recuperado de: $\quad$ https://rua.ua.es/dspace/ bitstream/10045/13540/1/ALT_16_01. pdf

Gamboa, A. A., Hernández, C. A, \& Prada, R. (2021). Efectos depresivos del aislamiento preventivo obligatorio asociados a la pandemia del COVID-19 en docentes y estudiantes de una universidad pública en Colombia. Psicogente, 24(45), 1-20. Doi: https://doi.org/10.17081/ psico.24.45.4156

Hernández, C., Gamboa, A. A., \& Prada, R. (2021). Competencias de maestros de ciencias naturales: una lectura desde las prácticas pedagógicas. Revista Boletín Redipe, 10(3), 360-375.Doi: https://doi. org/10.36260/rbr.v10i3.1240

Hernández Vergel, V. K., Prada González, M. P., Villabona, B. J., \& Prada Núñez, R. (2020). Diagnóstico del uso de las redes sociales por estudiantes de educación básica secundaria y su posible uso educativo. Seminario Internacional de Práctica Pedagógica, 1292.

Ibáñez, J. S. (2020). Educación en tiempos de pandemia: tecnologías digitales en la mejora de los procesos educativos. Innovaciones educativas, 22(Especial), 1721. Doi: https://doi.org/10.22458/ ie.v22iEspecial.3173
Laurente, C. M., Rengifo, R. A., Asmat, N. S. y Neyra, L. (2020). Desarrollo de competencias digitales en docentes universitarios a través de entornos virtuales. Revista Eleuthera, 22(2), 7187. Doi: 10.17151/eleu.2020.22.2.5.

López-García, J. D., \& Gutiérrez-Niño, D. (2018). Efecto del uso de la herramienta "realidad aumentada" en el rendimiento académico de estudiantes de Educación Básica. Revista Perspectivas, 3(1), 6-12. https://doi.org/10.22463/25909215.1464

Martín, M. M., Hernández-Suarez, C. A., \& Mendoza-Lizcano, S. M. (2017). Ambientes de aprendizaje basados en herramientas web para el desarrollo de competencias TICenladocencia. Revista Perspectivas, 2(1), 97-104. https://doi. org/10.22463/25909215.1282

Mas, Ó., y Olmos, P. (2016). El profesor universitario en el Espacio Europeo de Educación Superior: la autopercepción de sus competencias docentes actuales y orientaciones para su formación pedagógica. Revista mexicana de investigación educativa, 21(69), 437470. Recuperado de: http://www.scielo. org. $\mathrm{mx} / \mathrm{pdf} / \mathrm{rmie} / \mathrm{v} 21 \mathrm{n69} / 1405-6666$ rmie-21-69-00437.pdf

Montgomery, D.C., \& Runger, G.C. (2010). Applied Statistics and Probability for Engineers. John Wiley \& Sons.

Penagos, M., Mariño, L. F., \& Hernández, R. V. (2017). Pensamiento matemático elemental y avanzado como actividad humana en permanente evolución. Revista Perspectivas, 2(1), 105-116. https://doi.org/10.22463/25909215.1289

Prada, R., Gamboa, A. A., \& Hernández, C. A. (2021). Conocimiento didácticomatemático en docentes de básica y media. Revista Boletín Redipe, 10(6), 259-273. Doi: https://doi.org/10.36260/ rbr.v10i6.1323 
Rambay, M., y De la Cruz, J. (2021). Desarrollo de las Competencias Digitales en los Docentes Universitarios en Tiempo Pandemia: Una Revisión Sistemática. In Crescendo, 11(4), 511-527. Recuperado de: https://revistas.uladech.edu. pe/index.php/increscendo/article/ view/2331

Rodríguez Zambrano, Hernando (2007). El paradigma de las competencias hacia la educación superior. Revista de la Facultad de Ciencias Económicas: Investigación y Reflexión, XV (1), 145165. Recuperado de: https://www. redalyc.org/articulo.oa?id=90915108

Ruiz, M. P. (2006). Las competencias, instrumento para un cambio de paradigma. In Investigación en educación matemática: actas del $X$ Simposio de la Sociedad Española de Investigación en Educación Matemática, Huesca, 6-9 de septiembre de 2006. 83-106. Instituto de Estudios Altoaragoneses. Recuperado de: Dialnet-LasCompeten ciasInstrumentoParaUnCambioDePara digma-2264720.pdf

Sandí, J. C., y Sanz, C. V. (2018). Revisión y análisis sobre competencias tecnológicas esperadas en el profesorado en Iberoamérica. Edutec. Revista Electrónica De Tecnología Educativa, (66), 93-121. Doi: https://doi. org/10.21556/edutec.2018.66.1225

Sesento, L. (2008). Modelo Sistémico Basado en Competencias para Instituciones Educativas Públicas. (Tesis de grado). Centro de Investigación y Desarrollo en el estado de Michoacán - CIDEM. Morelia, Michoacán.

Tejada, J., y Pozos, K. (2018). Nuevos escenarios y competencias digitales docentes: hacia la profesionalización docente con TIC. Profesorado, revista de currículum y formación del profesorado, 22(1), 25-51. Doi: https://doi.org/10.30827/ profesorado.v22i1.9917

Vargas, L. V., Gamboa, A. A., \& Prada, R. (2021). Competencias lecto-escritoras en educación media: Deconstrucción, Reconstrucción y Evaluación de la práctica Pedagógica. Revista Boletín Redipe, 10(6), 356-365. Doi: https://doi. org/10.36260/rbr.v10i6.1331

Villamizar-Acevedo, G. A., Lozano-León, S. G., \& Sierra-Garavito, E. D. (2017). Creencias sobre las fuentes y formas de acceso al conocimiento generadas en las prácticas pedagógicas desde la perspectiva del estudiante. Revista Perspectivas, 2(1), 18-27. https://doi. org/10.22463/25909215.1281

Zabalza, M. Á. (2003). Competencias docentes del profesorado universitario: calidad y desarrollo profesional. Narcea S.A. ediciones: Madrid, España. 